\title{
Unilateral Fimbria/Fornix Transection Prevents the Synaptoplastic Effect of Dehydroepiandrosterone in the Hippocampus of Female, but Not Male, Rats
}

\author{
Ari L. Mendell ${ }^{1}$, Neil J. MacLusky ${ }^{1 *}$, Csaba Leranth ${ }^{2,3}$ \\ ${ }^{1}$ Department of Biomedical Sciences, Ontario Veterinary College, University of Guelph, Guelph, Canada; ${ }^{2}$ Department of Obstetrics, \\ Gynecology \& Reproductive Sciences, Yale University School of Medicine, New Haven, USA; ${ }^{3}$ Department of Neurobiology, Yale \\ University School of Medicine, New Haven, USA. \\ Email: *neil.maclusky@gmail.com
}

Received May $29^{\text {th }}, 2013$; revised June $25^{\text {th }}, 2013$; accepted July $4^{\text {th }}, 2013$

Copyright (C 2013 Ari L. Mendell et al. This is an open access article distributed under the Creative Commons Attribution License, which permits unrestricted use, distribution, and reproduction in any medium, provided the original work is properly cited.

\begin{abstract}
Dehydroepiandrosterone (DHEA), the most abundant adrenal androgen in primates, is also synthesized from cholesterol in the brain. Like testosterone, DHEA induces spine synapse formation in the hippocampus. In female rats, this response is blocked by co-administration of an inhibitor of aromatase, the enzyme responsible for estrogen biosynthesis. In males, by contrast, the hippocampal synaptic response to DHEA is unaffected by treatment with an aromatase inhibitor. We hypothesized that this sex difference might reflect differential dependence of the hippocampal responses on subcortical afferents from the basal forebrain. To test this hypothesis, we examined the effects of unilateral fimbria/ fornix transection (FFX) on DHEA-induced synapse formation in the cornu ammonis 1 (CA1) hippocampal subfield of gonadectomized female and male rats. In ovariectomized females, CA1 spine synapse density after DHEA treatment was reduced by more than 60\% ipsilateral to FFX. In males, however, unilateral FFX transection had no effect on spine synapse density after DHEA treatment. These results suggest that sex differences in the dependence on local estrogen biosynthesis of the CA1 synaptic response to androgen may at least in part be the result of sex differences in the relative contributions of afferents to the hippocampus from the basal forebrain.
\end{abstract}

Keywords: Dehydroepiandrosterone; Subcortical Mediation; Synaptic Plasticity; Hippocampus; Sex Differences

\section{Introduction}

Gonadal hormones play an essential role in the maintenance of spine synapses in the CA1 subfield of the hippocampus. Both estrogens [1-4] and androgens [5] regulate hippocampal spine synapse density in female rats, whereas only androgens regulate synapse density in males [6]. The mechanisms by which gonadal hormones exert their actions on synapses in the hippocampus are incompletely characterized.

Dehydroepiandrosterone (DHEA) is the most abundant circulating androgen produced by the adrenal glands in primates, and there is a significant decline in DHEA levels with age $[7,8]$. A number of studies have indicated that DHEA is also synthesized in the CNS [9-11] where it may have direct effects on neuronal activity [12]. Although it has relatively weak androgenic activity in non-

"Corresponding author. neural androgen target tissues, DHEA has been reported to have positive effects on cognitive function [13], as well as mood [14]. It has also been demonstrated that DHEA has the ability to increase hippocampal spine synapse density in gonadectomized female [15] and male rats [16]. The mechanisms underlying these effects remain poorly understood.

In females, the synaptic effects of DHEA are blocked by the aromatase inhibitor letrozole [15]. This suggests that DHEA effects in the female hippocampus are similar to those of testosterone, in which they are dependent on conversion to estrogen [17]. In gonadectomized male rats, however, letrozole has no effect on DHEA-induced increases in hippocampal spine synapses [16], indicating that there are sex differences in the mechanisms by which these responses are produced.

Previous reports have shown that in females the effects of estrogen $[2,18]$ on the hippocampus are dependent on 
input from subcortical cholinergic neurons. Thus, transection of the fimbria/fornix (FF) [2] or destruction of cholinergic neurons in the medial septum and diagonal band of Broca (MSDB) by intraventricular injection of 192 IgG-saporin [19] abolish the effects of estrogen on CA1 spine synapse density. The MSDB region of the brain contains high concentrations of aromatase [20]. We hypothesized that the sex difference in the dependence on aromatase activity of the hippocampal synaptic effects of DHEA might at least in part be due to differences between males and females in the relative contributions from subcortical input. If so, we might expect a profound sex difference in the effects of eliminating this input on the hippocampal synaptic response to DHEA. To test this hypothesis, we examined the effect of unilateral fimbria/ fornix transection (FFX) on the effects of DHEA treatment on hippocampal synaptic plasticity, in gonadectomized male and female rats.

\section{Materials and Methods}

\subsection{Animal Subjects}

Adult male and female Sprague Dawley rats (60 - 70 days old) were obtained from Charles River, Wilmington, MA, USA. Animals were maintained in individual cages with water and regular rat chow available ad libitum, and kept on a 12-hour light/dark cycle. All experiments included as a part of this study conformed to relevant University Animal Care and Use Committee guidelines and were in compliance with both the National Institutes of Health Guide for the Care and Use of Laboratory Animals and the guidelines of the Canadian Council of Animal Care.

\subsection{Surgical Procedures and Hormone Treatment}

Three male rats and three female rats were included in this study. All rats were anesthetized for surgery using a ketamine cocktail [3 ml/kg, i.m.; ketamine $(25 \mathrm{mg} / \mathrm{ml})+$ xylazine $(1.2 \mathrm{mg} / \mathrm{ml})+$ acepromazine $(0.03 \mathrm{mg} / \mathrm{ml})$ in saline $(0.9 \%)]$.

The female rats were ovariectomized via small dorsal flank incisions, and all males were orchidectomized. All animals then underwent surgical unilateral transection of the fimbria/fornix, as previously described $[2,17]$. Briefly, the animals were fixed in a stereotaxic apparatus (David Kopf Instruments, Tujunga, CA, USA), and, following dorsal penetration and subsequent aspiration of the overlying cortical areas and corpus callosum, the right fimbria/fornix was completely transected under visual control. One week after surgery, all female and male rats received DHEA treatment (Sigma Chemical Co. St. Louis, MO), given in the form of two subcutaneous injections ( $1 \mathrm{mg} /$ day, in sesame oil) separated by 24 hours.
For this study, the intact side of the brain, contralateral to fimbria/fornix transection, was used as the control. It has previously been shown that DHEA administration induces spine synapses in both males and females to levels indistinguishable from intact controls [15,16]. It has also been shown that unilateral transection of the fimbria/ fornix has no significant effect on the synaptic response to testosterone of the contralateral hippocampus [17]. Hence the response contralateral to FFX can be used as a matched, individual control for the effects of the steroid, in each animal. In the present study, the average spine synapse density we observed for female rats contralateral to FFX very closely matched previous observations of ovariectomized females treated with DHEA [16] or testosterone [5], while the spine synapse density numbers we observed ipsilateral to FFX were comparable, but slightly lower than previously published density values for ovariectomized females $[2,5,16]$. For the males, the spine synapse density values on both sides of the hippocampus very closely matched previously published reports of spine synapse density in either intact males, or orchidectomized males following DHEA [16] or testosterone [6] treatment.

\subsection{Tissue Processing}

Two days following the second injection of DHEA, rats were killed under deep ether anesthesia by transcardial perfusion of heparinized saline, followed by a fixative containing $4 \%$ paraformaldehyde and $0.1 \%$ glutaraldehyde in $0.1 \mathrm{M}$ phosphate buffer ( $\mathrm{pH}$ 7.35). The descending aorta was firmly clamped for the course of the perfusion, in order to prevent access of the fixative to the lower half of the body. Brains were removed and postfixed overnight in the same fixative used for perfusion, without the glutaraldehyde. The hippocampi were dissected out, and coronal vibratome sections $(100 \mu \mathrm{m})$ were cut. The sections were then postfixed in $1 \%$ osmium tetroxide (30 min), dehydrated in ethanol (the $70 \%$ ethanol contained $1 \%$ uranyl acetate) for $30 \mathrm{~min}$, and flat embedded in Araldite.

\subsection{Synapse Counts}

Spine synapse density was calculated using unbiased stereological methods, as previously described $[2,15,16]$. Briefly, in order to assess any potential changes in the volume of the tissue, a correction factor was first calculated, assuming that the treatment and surgical procedures had no effect on the total number of pyramidal cells in the hippocampus [16,21]. In all hippocampi, six or seven disector pairs (pairs of adjacent $2 \mu \mathrm{m}$ toluidine blue-stained semithin sections mounted on slides) were analyzed using the technique of Braendgaard and Gundersen [22]. The cell density value (D) for pyramidal 
cells was calculated using the formula $D=N / s T$, where $\mathrm{N}$ is the mean disector score across all sampling windows, $\mathrm{T}$ is the thickness of the sections $(2 \mu \mathrm{m})$, and $\mathrm{s}$ is the unit area of the window. Based on these values, a dimensionless volume correction factor $\left(\mathrm{k}_{\mathrm{v}}\right)$ was introduced: $\mathrm{k}_{\mathrm{v}}$ $=\mathrm{D} / \mathrm{D}_{1}$, where $\mathrm{D}_{1}$ is the mean pyramidal cell density across the groups of hippocampi.

Thereafter, pairs of consecutive serial ultrathin sections (reference and look-up) were cut from the Araldite-embedded vibratome sections, from an area located between the middle and upper third of the stratum radiatum of CA1 (300 - $500 \mu \mathrm{m}$ from the pyramidal cell layer; for precise location, see [5]), and collected on Formvarcoated single-slot grids. Digitized images were taken at a magnification of $\times 9900$ using a Tecnai 12 transmission electron microscope furnished with an AMT Advantage 4.00 HR/HR-B CCD camera system, with the observer blinded to the experimental treatment. At least five neuropil fields were photographed on each electron microscopic grid. With at least eight grids from each vibratome section (five vibratome sections from each hippocampus taken from different areas along the septo-temporal axis) containing a minimum of two pairs of consecutive, serial ultrathin sections, each animal provided a minimum of 5 $\times 8 \times 5=200$ or more neuropil fields. Identical regions in reference and look-up sections were identified using easily recognizable landmarks such as large myelinated fibers, large dendrites or blood vessels with distinct orientations, which were not changed significantly between neighbouring sections. Areas occupied by potentially interfering structures such as large dendrites, glial cells, or capillaries were avoided, or subtracted from measured areas using the NIH Scion Image software.

In order to obtain a comparable number of synaptic numbers, unbiased for possible changes in synapse size, the disector technique was used [23]. The digitized electron micrographs were printed using a laser printer, and a reference grid superimposed onto the micrographs was used to aid in calculation of spine synapse density. The disector volume (volume of reference used for density calculations) was the unit area of the reference grid multiplied by the distance between the upper faces of the reference and look-up sections [22]. Section thickness (average $0.075 \mu \mathrm{m}$ ) was determined using the electron scattering technique [24]. The calculated spine synapse density values were then divided by the $\mathrm{k}_{\mathrm{v}}$. This correction provided a normalized spine synapse density, accounting for the density of pyramidal cells and for any changes in hippocampal volume. Prior to data analysis, the printed pictures were coded to ensure blind synapse counting, and this code was not broken until the analysis was completed. Only those spine synapses that were present in the reference section but not in the look-up section, or vice versa, were counted [4].

\subsection{Statistical Analysis}

Spine synapse density counts were averaged for each animal on each side of the hippocampus, and an overall mean was then taken for each group. Values are presented as mean \pm S.E.M. Two-way ANOVA was used to statistically analyze the data, followed by unequal variance, two-tailed t-tests for comparison of individual group means. All statistical tests were performed using Statview statistical software (SAS institute, Cary, NC, USA), and statistical significance was set to $\mathrm{p}<0.05$ (two-tailed).

\section{Results}

The effect of DHEA on spine synapse density in the stratum radiatum of CA1 following unilateral FFX in females and males is demonstrated in Figure 1. Two-
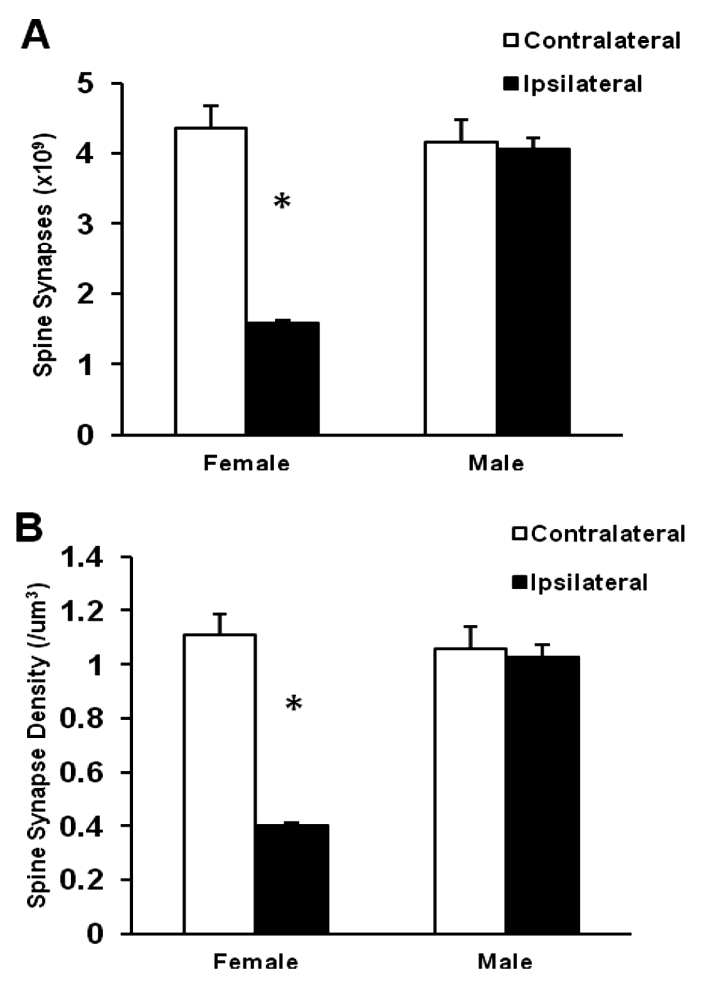

Figure 1. Spine synapses in gonadectomized female and male rats. Bar graphs show the number of spine synapses (A) and the spine synapse density (B) in the CA1 stratum radiatum of gonadectomized, unilaterally fimbria/fornix transected (FFX), dehydroepiandrosterone (DHEA)-treated female and male rats. DHEA treatment increased spine synapses to normal and approximately equal levels in both the contralateral and ipsilateral sides of the hippocampus to FFX in male rats, and the contralateral side to FFX in the females. The spine synapse density in the ipsilateral side to FFX in females was not increased by DHEA treatment, and was significantly lower $(\sim 63.8 \%)$ compared to the contralateral side [ ${ }^{*}$ represents $P<0.05$. Bars represent mean \pm S.E.M.]. 
way ANOVA showed significant differences in synapse density caused by FFX side $(F=35.863, p=0.0003)$ and sex $(F=22.261, p=0.0015)$, as well as a significant interaction effect of FFX side and sex $(F=30.426, p=$ 0.0006). In ovariectomized females, the number of CA1 spine synapses ipsilateral to FFX was significantly lower (63.8\%; t-test: $\mathrm{p}=0.012 ; 1.577 \times 10^{9} \pm 0.043 \times 10^{9}$ synapses) than the contralateral side $\left(4.361 \times 10^{9} \pm 0.322 \times\right.$ $10^{9}$ synapses) following DHEA treatment. For orchidectomized males receiving the same surgical procedure and DHEA treatment, the numbers of spine synapses on both sides of the hippocampus were indistinguishable, with no significant difference observed (t-test: $\mathrm{p}=0.771 ; 4.167 \times$ $10^{9} \pm 0.319 \times 10^{9}$ on the contralateral side; $4.053 \times 10^{9} \pm$ $0.164 \times 10^{9}$ on the ipsilateral side).

\section{Discussion}

These observations expand previous findings showing that DHEA treatment increases hippocampal spine synapse density, in both male [16] and female rats [15]. Here, we demonstrate that the positive effect of DHEA on spine synapses in hippocampal area CA1 in females is dependent on afferents from subcortical structures via the FF pathway. It is well established that estrogen increases the density of hippocampal spine synapses in female rats [1,3-5] as well as non-human primates [25,26]. In rats, these responses are highly de- pendent on afferents from estrogen-sensitive subcortical structures, including the supramammillary area, MSDB and median raphe nuclei $[2,18]$. Androgens also promote synaptogenic responses in female rats, but the effects of both testosterone and DHEA in females are almost completely blocked by pretreatment with an aromatase inhibitor [5,15]. Together with the observations from the present study, these results suggest that the synaptic effect of DHEA in the female hippocampus may occur primarily through aromatization of DHEA in the brain and effects of estrogen that require intact subcortical afferents arriving in the hippocampus via the FF.

Previous work has demonstrated that the dose of DHEA used here (1 $\mathrm{mg} / \mathrm{rat}$ s.c., daily for 2 days) promotes full recovery of spine synapses in the stratum radiatum of CA1 of the hippocampus in orchidectomized male rats, even though in males estradiol has no significant effect on hippocampal spine synapse density $[6,16]$. The present data show that, in contrast to the situation in females, the response to DHEA is unaffected by unilateral transection of the fimbria-fornix, suggesting that the mechanism by which DHEA produces its synaptic effects in males not only does not involve intermediate estrogen biosynthesis, it is also independent of subcortical mediation.

One hypothesis to explain these findings might be that, in males, the actions of androgens on synapse density are mediated locally, within the hippocampus itself, while in females these responses involve a much larger contribution from estrogen-sensitive basal forebrain cholinergic neurons. While such a model would be consistent with the present data, other studies suggest that additional factors are probably also involved. A large body of evidence suggests that at least some of the effects of estradiol on hippocampal synaptic plasticity in females are mediated via estrogen receptors located within the hippocampus itself [27-29]. Previous work has demonstrated that the synaptic effects of testosterone are partially blocked by FFX in male rats [17]. Thus, at least for testosterone, some of the synaptic effects in males appear to involve sub-cortical afferent input. Testosterone is known to regulate the basal forebrain cholinergic system: thus, in orchidectomized males testosterone, but not estradiol, increases choline acetyltransferase activity in the medial preoptic area [30]. The absence of a response to estradiol in males could potentially represent contributions from both sexual differentiation of the responses to estradiol of the basal forebrain cholinergic system and the lack of local, rapid signaling effects of estradiol in the male hippocampus [27,28,31]. Conversely, in males testosterone could modulate hippocampal synaptic plasticity via both modulation of afferent cholinergic input [17], and effects mediated via local membrane androgen receptors, which are present throughout the hippocampus $[32,33]$ and have been localized to pre-terminal axons, axon terminals, and dendritic spines in CA1 [33]. Such a mechanism would be consistent with the observation that testosterone induced increases in hippocampal spine synapse density are partially blocked by FF transection [17].

Why then are the effects of DHEA in males unaffected by FFX? There are two possible explanations. Since the androgenic potency of DHEA is lower than that of testosterone, its effects on the basal forebrain cholinergic system may be weaker than those of testosterone, which could explain why the hippocampal synaptic response to DHEA is unaffected by FFX. Alternatively, DHEA and testosterone may exert their effects via different receptor mechanisms. Several studies have suggested that DHEA and its sulfate conjugate can act on the hippocampus via receptors distinct from those mediating the actions of testosterone and estradiol [34], including direct effects on mGluR5 metabotropic glutamate receptors in the hippocampus [34]. If such mechanisms are primarily responsible for mediating the synaptic effects of DHEA in males, this could explain why the response to DHEA in males is unaffected by FF transection. Further work on the relative effects of DHEA on different components of the basal forebrain-hippocampal circuitry will be required to test this hypothesis. 


\section{Conclusion}

This study demonstrates that the effects of DHEA on spine synapses in the CA1 subfield of the hippocampus may involve different mechanisms in female and male rats. The effects of the weak androgen DHEA on CA1 synapse density in the male are unaffected by FF transection, whereas the effects of this steroid on the same region of the female are largely dependent on subcortical mediation. These findings highlight sex differences in the importance of afferent input from subcortical structures in regulating hippocampal synaptic plasticity

\section{Acknowledgements}

This work was supported by Natural Sciences and Engineering Research Council of Canada Discovery Grant 197293-2007 (NJM) and National Institutes of Health Grant ES017013 (CL). ALM is a recipient of an Ontario Graduate Studentship.

\section{REFERENCES}

[1] T. Hajszan, K. Szigeti-Buck, N. L. Sallam, J. Bober, A. Parducz, N. J. MacLusky, C. Leranth and R. S. Duman, "Effects of Estradiol on Learned Helplessness and Associated Remodeling of Hippocampal Spine Synapses in Female Rats,” Biological Psychiatry, Vol. 67, No. 2, 2010, pp. 168-174. doi:10.1016/j.biopsych.2009.08.017

[2] C. Leranth, M. Shanabrough and T. L. Horvath, "Hormonal Regulation of Hippocampal Spine Synapse Density Involves Subcortical Mediation,” Neuroscience, Vol. 101, No. 2, 2000, pp. 349-356.

doi:10.1016/S0306-4522(00)00369-9

[3] C. S. Woolley, "Estrogen-mediated Structural and Functional Synaptic Plasticity in the Female Rat Hippocampus," Hormones and Behavior, Vol. 34, No. 2, 1998, pp. 140-148. doi:10.1006/hbeh.1998.1466

[4] C. S. Woolley and B. S. McEwen, "Estradiol Mediates Fluctuation in Hippocampal Synapse Density during the Estrous Cycle in the Adult Rat," Journal of Neuroscience, Vol. 12, No. 7, 1992, pp. 2549-2554.

[5] C. Leranth, T. Hajszan and N. J. MacLusky, “Androgens Increase Spine Synapse Density in the CA1 Hippocampal Subfield of Ovariectomized Female Rats,” Journal of Neuroscience, Vol. 24, No. 2, 2004, pp. 495-499. doi:10.1523/JNEUROSCI.4516-03.2004

[6] C. Leranth, O. Petnehazy and N. J. MacLusky, "Gonadal Hormones Affect Spine Synaptic Density in the CA1 Hippocampal Subfield of Male Rats,” Journal of Neuroscience, Vol. 23, 2003, pp. 1588-1592.

[7] S. G. Beck and R. J. Handa, "Dehydroepiandrosterone (DHEA): A Misunderstood Adrenal Hormone and Spinetingling Neurosteroid,” Endocrinology, Vol. 145, No. 3, 2004, pp. 1039-1041. doi:10.1210/en.2003-1703

[8] F. Labrie, A. Belanger, L. Cusan, J. L. Gomez and B. Candas, "Marked Decline in Serum Concentrations of
Adrenal C19 Sex Steroid Precursors and Conjugated Androgen Metabolites during Aging," Journal of Clinical Endocrinology and Metabolism, Vol. 82, No. 8, 1997, pp. 2396-2402. doi:10.1210/jc.82.8.2396

[9] E. E. Baulieu, "Steroid Hormones in the Brain: Several Mechanisms?” In: K. Fuxe, J. A. Gutafsson and L. Wetterberg, Eds., Steroid Hormone Regulation of the Brain, Pergamon Press, Oxford, 1981, pp. 3-14.

[10] E. E. Baulieu and P. Robel, "Dehydroepiandrosterone (DHEA) and Dehydroepiandrosterone Sulfate (DHEAS) as Neuroactive Neurosteroids," Proceedings of the $\mathrm{Na}$ tional Academy of Sciences of USA, Vol. 95, No. 8, 1998, pp. 4089-4091. doi:10.1073/pnas.95.8.4089

[11] C. Le Goascogne, P. Robel, M. Gouézou, N. Sananès, E. E. Baulieu and M. Waterman, "Neurosteroids: Cytochrome P450scc in Rat Brain,” Science, Vol. 237, No. 4819, 1987, pp. 1212-1215. doi:10.1126/science.3306919

[12] P. Zheng, "Neuroactive Steroid Regulation of Neurotransmitter Release in the CNS: Action, Mechanism and Possible Significance,” Progress in Neurobiology, Vol. 89, No. 2, 2009, pp. 134-152. doi:10.1016/j.pneurobio.2009.07.001

[13] M. Vallee, W. Mayo and M. Le Moal, "Role of Pregnenolone, Dehydroepiandrosterone and Their Sulfate Esters on Learning and Memory in Cognitive Aging," Brain Research Reviews, Vol. 37, No. 1-3, 2001, pp. 301-312. doi:10.1016/S0165-0173(01)00135-7

[14] R. K. Sripeda, C. E. Marx, A. P. King, N. Rajaram, S. N. Garfinkel, J. L. Abelson and I. Liberzon, "DHEA Enhances Emotion Regulation Neurocircuits and Modulates Memory for Emotional Stimuli," Neuropsychopharmacology. doi:10.1038/npp.2013.79

[15] T. Hajszan, N. J. MacLusky and C. Leranth, "Dehydroepiandrosterone Increases Hippocampal Spine Synapse Density in Ovariectomized Female Rats,” Endocrinology, Vol. 145, No. 3, 2004, pp. 1042-1045. doi:10.1210/en.2003-1252

[16] N. J. MacLusky, T. Hajszan and C. Leranth, "Effects of Dehydroepiandrosterone and Flutamide on Hippocampal CA1 Spine Synapse Density in Male and Female Rats: Implications for the Role of Androgens in Maintenance of Hippocampal Structure,” Endocrinology, Vol. 145, No. 9, 2004, pp. 4154-4161. doi:10.1210/en.2004-0477

[17] E. G. Kovacs, N. J. MacLusky and C. Leranth, “Effects of Testosterone on Hippocampal CA1 Spine Synaptic Density in the Male Rat are Inhibited by Fimbria/Fornix Transection,” Neuroscience, Vol. 122, No. 3, 2003, pp. 807-810. doi:10.1016/j.neuroscience.2003.08.046

[18] C. Leranth and M. Shanabrough, "Supramamillary Area Mediates Subcortical Estrogenic Action on Hippocampal Synaptic Plasticity,” Experimental Neurology, Vol. 167, No. 2, 2001, pp. 445-450. doi:10.1006/exnr.2000.7585

[19] T. T. Lam and C. Leranth, "Role of the Medial Septum Diagonal Band of Broca Cholinergic Neurons in Oestrogen-Induced Spine Synapse Formation on Hippocampal CA1 Pyramidal Cells of Female Rats,” European Journal of Neuroscience, Vol. 17, No. 10, 2003, pp. 1997-2005. doi:10.1046/j.1460-9568.2003.02637.x 
[20] T. L. Horvath, L. Roa-Pena, R. L. Jakab, E. R. Simpson and F. Naftolin, “Aromatase in Axonal Processes of Early Postnatal Hypothalamic and Limbic Areas Including the Cingulate Cortex," Journal of Steroid Biochemistry and Molecular Biology, Vol. 61, No. 3-6, 1997, pp. 349-357. doi:10.1016/S0960-0760(97)80032-5

[21] D. A. Rusakov, H. A. Davies, E. Harrison, G. Diana, G. Richter-Levin, T. V. P. Bliss and M. G. Stewart, "Ultrastructural Synaptic Correlates of Spatial Learning in Rat Hippocampus,” Neuroscience, Vol. 80, No. 1, 1997, pp. 69-77. doi:10.1016/S0306-4522(97)00125-5

[22] H. Braendgaard and H. J. Gundersen, “The Impact of Recent Stereological Advances on Quantitative Studies of the Nervous System," Journal of Neuroscience Methods, Vol. 18, No. 1-2, 1986, pp. 39-78. doi:10.1016/0165-0270(86)90112-3

[23] D. C. Sterio, "The Unbiased Estimation of Number and Sizes of Arbitrary Particles Using the Disector,” Journal of Microscopy, Vol. 134, No. 2, 1984, pp. 127-136. doi:10.1111/j.1365-2818.1984.tb02501.x

[24] J. V. Small, “Measurement of Section Thickness,” In: D.S. Bocciarelli, Ed., Proceedings of the 4th European Conference on Electron Microscopy, Tipographia Poliglotta Vatican, Rome, 1968, pp. 609-610.

[25] C. Leranth, M. Shanabrough and D. E. Redmond Jr., "Gonadal Hormones are Responsible for Maintaining the Integrity of Spine Synapses in the CA1 Hippocampal Subfield of Female Non-human Primates,” Journal of Comparative Neurology, Vol. 447, No. 1, 2002, pp. 34-42. doi:10.1002/cne.10230

[26] C. Leranth, T. Hajszan, K. Szigeti-Buck, J. Bober and N. J. MacLusky, "Bisphenol A Prevents the Synaptogenic Response to Estradiol in Hippocampus and Prefrontal Cortex of Ovariectomized Nonhuman Primates," Proceedings of the National Academy of Sciences, Vol. 105, No. 37, 2008, pp. 14187-14191. doi:10.1073/pnas.0806139105

[27] M. I. Boulware, J. P. Weick, B. R. Becklund, S. P. Kuo, R. D. Groth and P. G. Mermelstein, "Estradiol Activates Group I and II Metabotropic Glutamate Receptor Signaling, Leading to Opposing Influences on cAMP Response Element-Binding Protein,” Journal of Neuroscience, Vol.
25, No. 20, 2005, pp. 5066-5078. doi:10.1523/JNEUROSCI.1427-05.2005

[28] M. I. Boulware, H. Kordasiewicz and P. G. Mermelstein, "Caveolin Proteins Are Essential for Distinct Effects of Membrane Estrogen Receptors in Neurons,” Journal of Neuroscience, Vol. 27, No. 37, 2007, pp. 9941-9950. doi:10.1523/JNEUROSCI.1647-07.2007

[29] D. P. Srivastava, E. M. Waters, P. G. Mermelstein, E. A. Kramar, T. J. Shors and F. Liu, "Rapid Estrogen Signaling in the Brain: Implications for the Fine-Tuning of Neuronal Circuitry,” Journal of Neuroscience, Vol. 31, No. 35, 2011, pp. 16056-16063. doi:10.1523/JNEUROSCI.4097-11.2011

[30] V. N. Luine, R. I. Khylchevskaya and B. S. McEwen, "Effect of Gonadal Steroids on Activities of Monoamine Oxidase and Choline Acetylase in Rat Brain,” Brain Research, Vol. 86, No. 2, 1975, pp. 293-306. doi:10.1016/0006-8993(75)90704-0

[31] J. Meitzen, D. D. Grove and P. G. Mermelstein, “The Organizational and Aromatization Hypotheses Apply to Rapid, Nonclassical Hormone Action: Neonatal Masculinization Eliminates Rapid Estradiol Action in Female Hippocampal Neurons,” Endocrinology, Vol. 153, No. 10, 2012, pp. 4616-21. doi:10.1210/en.2012-1525

[32] S. Sarkey, I. Azcoitia, L. M. Garcia-Segura, D. GarciaOvejero and L. L. DonCarlos, "Classical Androgen Receptors in Nonclassical Sites in the Brain,” Hormones and Behavior, Vol. 53, No. 5, 2008, pp. 753-764. doi:10.1016/j.yhbeh.2008.02.015

[33] N. E. Tabori, L. S. Stewart, V. Znamensky, R. D. Romeo, S. E. Alves, B. S. McEwen and T. A. Milner, "Ultrastructural Evidence That Androgen Receptors Are Located at Extranuclear Sites in the Rat Hippocampal Formation," Neuroscience, Vol. 130, No. 1, 2005, pp. 151163. doi:10.1016/j.neuroscience.2004.08.048

[34] Y. Xu, M. Tanaka, L. Chen and M. Sokabe, "DHEAS Induces Short-Term Potentiation via the Activation of a Metabotropic Glutamate Receptor in the Rat Hippocampus,” Hippocampus, Vol. 22, No. 4, 2012, pp. 707-722. doi:10.1002/hipo.20932 